\title{
Reinstating Soil Biodiversity: The Key for Converting Degraded Lands to Sustainable Systems
}

\author{
Wijepala P.C. ${ }^{1 *}$, Seneviratne G. ${ }^{1}$, Madawala H.M.S.P. ${ }^{2}$ and Jayasuriya K.M.G.G. ${ }^{2}$ \\ ${ }^{I}$ National Institute of Fundamental Studies, Sri Lanka \\ ${ }^{2}$ Department of Botany, University of Peradeniya, Sri Lanka \\ *piyumichathurika8136@gmail.com
}

\begin{abstract}
Lost soil biodiversity in croplands can be reinstated by using microbial biotechnological methods. Conventional agricultural practices collapse diversity of soil microbial communities. This may lead to decline in crop productivity and sustainability. Direct application of developed fungal-bacterial communities known as biofilmed biofertilizers (BFBFs) has been introduced recently, which reduces the chemical fertilizer usage with other crop and soil benefits over conventional biofertilizers. This study investigates the effect of BFBFs on microbial diversity and introduces them as a remedial measure to recover degraded croplands. A degraded tea soil was separated in to particle size fractions and transferred to trays that were arranged in a completely randomized design with three replicates in a greenhouse. Biofilm-T, the BFBF developed for tea was used with a control (distilled water). Certain soil biological and chemical properties were measured after three months of incubation. Application of Biofilm- $\mathrm{T}$ significantly increased $\mathrm{pH}$ in fine fraction. Available nutrient contents varied in the soil fractions. Furthermore, addition of Biofilm-T significantly increased bacterial abundance in clay fraction due to dormancy breaking of microbial seed bank by the BFBF-specific biomolecules. Total carbon and nitrogen contents were significantly high in fine and clay fractions due to presence of higher algal and cyanobacterial growth. This study concludes that application of BFBFs breaks dormancy of microbial seed bank trapped in different soil fractions and also assists in improving nutrient release from them, thus reinstating soil biodiversity and sustainability of degraded croplands.
\end{abstract}

Keywords: Agriculture, Biofilm biofertilzer, Micorbial biofilms, Microbial diversity, Soil health 